\title{
TRATAMIENTO MULTIDISCIPLINARIO ACTUAL DEL CÁNCER DE PRÓSTATA METASTÁSICO
}

\author{
M. RODRÍGUEZ CORDÓN*, E. FERRER ALBIACH*, C. FERRER ALBIACH** \\ Servicio de Oncología Radioterápica. *Hospital Clínico Universitario de Valencia. \\ **Hospital Provincial de Castellón. \\ Actas Urol Esp. 27 (10): 767-782, 2003
}

\section{RESUMEN}

TRATAMIENTO MULTIDISCIPLINARIO ACTUAL DEL CÁNCER DE PRÓSTATA METASTÁSICO

El cáncer de próstata es una causa común de muerte en hombres occidentales. La morbimortalidad asociada suele ser consecuencia directa de la diseminación ósea, presente en hasta un 50\% de los pacientes en el momento del diagnóstico. El objetivo del tratamiento de estos enfermos metastásicos es la prevención o paliación de los síntomas y de las complicaciones. El arsenal terapéutico es amplio e incluye hormonoterapia, radioterapia externa, radiofármacos, cirugía, quimioterapia, bisfosfonatos y nuevos fármacos (inhibidores de la angiogénesis, inmunoterapia y fármacos que actúan sobre la diferenciación celular). En la elección del tratamiento se deben considerar múltiples factores: la condición de andrógeno-dependiente o andrógeno-independiente, ya que la terapia hormonal constituye el primer escalón del tratamiento; la localización y extensión de la destrucción ósea; la gravedad del cuadro; la disponibilidad de las diferentes terapias; el estado del paciente; el pronóstico de supervivencia y la relación coste-efecto. Las indicaciones de algunas de estas terapias están claramente establecidas mientras que otras están todavía en estudio para determinar su eficacia, la pauta de tratamiento y las indicaciones. En este artículo se realiza una revisión y actualización de dichos tratamientos.

PALABRAS CLAVE: Próstata. Metastásico. Hormonoterapia. Radioterapia. Quimioterapia. Bisfosfonatos. Radiofármacos. Cirugía. Nuevas terapias.

\section{ABSTRACT \\ CURRENT MULTIAPPROACH TREATMENT OF METASTATIC PROSTATE CANCER}

Prostate cancer is one of the commonest causes of cancer-related death in the western world. The morbi-mortality associated is usually a direct consequence of metastatic spread to bone, in up to $50 \%$ of patients at first presentation. The aim of treatment of metastatic patients is to alleviate and to prevent the distressing symptoms. The approach include hormone-therapy, radiotherapy, radionuclides, surgery, chemotherapy, bisphosphonates and new drugs (agents that inhibit angiogenesis, inmmunotherapy and therapies that affect the differentiation). Decisions about therapy must also take into consideration the androgen-dependent or independent, so hormone-therapy is the first step of the treatment; the number and location of bone metastases; the severity of symptoms; the available of therapies; the status performance of patient; the prognosis and the cost-effect relationship. Some treatments have established indications whereas others are still in process of study in order to determinate their efficacy, their model of treatment and their indications. This article revises and updates these treatments. 
$\mathrm{E}^{1}$ cáncer de próstata es una causa común de muerte en hombres occidentales. En EE.UU, el riesgo de padecer la enfermedad es de un 9$11 \%$, habiéndose registrado en 1991 un total de 3.200 muertes secundarias a cáncer de próstata. En Europa, constituye la tercera causa de muerte relacionada con el cáncer en varones, tras el cáncer de pulmón y de colon. La morbimortalidad asociada a esta enfermedad suele ser consecuencia directa de la diseminación ósea, presente en hasta un $50 \%$ de los pacientes en el momento del diagnóstico. Por ello, se hace fundamental conocer las diferentes terapias disponibles para seleccionar la más adecuada a cada caso concreto.

\section{METÁSTASIS ÓSEAS EN EL CÁNCER DE PRÓSTATA}

Las metástasis óseas, sea cual sea su origen, actúan alterando la remodelación del hueso mediante la estimulación de los elementos celulares osteoclásticos y osteoblásticos, así como rompiendo los fibroblastos y los componentes hematógenos de la médula ósea ${ }^{1}$.

Las metástasis óseas de origen prostático se caracterizan por su actividad osteoblástica que parece estar relacionada con factores específicos estimulantes de la mitosis que se encuentran en el tejido prostático ${ }^{2}$. Existe también una reabsorción ósea acelerada que se ha podido demostrar mediante varios estudios histológicos y bioquímicos. El hueso destruido es sustituido por un tejido generado de forma anómala por la estimulación osteoblástica, siendo este hecho el responsable del aumento del volumen óseo y de la apariencia esclerótica de dichas lesiones en las radiografias ${ }^{3}$.

Las metástasis óseas pueden provocar fracaso de la médula ósea, hipercalcemia maligna, síndromes de compresión medular o de raíces nerviosas, fracturas patológicas y dolor. La causa del dolor no es bien conocida y probablemente influyen múltiples factores, siendo los estímulos mecánicos y químicos los más importantes, principalmente la distorsión y el estrés mecánico y prostaglandinas y leucotrienos que tienen un efecto estimulante de los receptores nociceptores del periostio ${ }^{4}$. El dolor es el síntoma más frecuente, estando presente en hasta un $84 \%$ de los pacientes con metástasis óseas en las fases ter- minales de la enfermedad. Por el contrario, la hipercalcemia maligna ${ }^{5}$ y las fracturas patológi$\operatorname{cas}^{6}$ tienen una incidencia más baja en las metástasis óseas de origen prostático que en otras enfermedades malignas.

Para realizar la elección del tratamiento en estos pacientes se deben considerar múltiples factores. Inicialmente, hay que clasificarlos en andrógeno-dependientes y andrógeno-independientes, hecho fundamental ya que la terapia hormonal constituye el primer escalón del tratamiento. Otros factores a tener en cuenta son la localización y extensión de la destrucción ósea, la gravedad del cuadro, la disponibilidad de las diferentes terapias, la existencia de tratamiento sistémico eficaz, el estado del paciente, el pronóstico de supervivencia y la relación coste-efecto.

\section{MODALIDADES DE TRATAMIENTO}

El objetivo del tratamiento de pacientes metastásicos no es, actualmente, la curación sino la prevención o paliación de los síntomas y de las complicaciones. El arsenal terapéutico disponible para el tratamiento de las neoplasias prostáticas en estadio extendido es amplio (Tabla I), pudiéndose emplear en un mismo paciente varias terapias, concomitante o secuencialmente, según los síntomas y el momento evolutivo de la enfermedad.

Las indicaciones de algunos de estos tratamientos están claramente establecidas, como ocurre con la hormonoterapia, la radioterapia externa o la terapia con estroncio. Otras están

\section{TABLA I}

MODALIDADES DE TRATAMIENTO

\begin{tabular}{||l|}
\hline 1. Terapia hormonal \\
\hline 2. Radioterapia externa \\
\hline 3. Quimioterapia \\
\hline 4. Bisfosfonatos \\
\hline \begin{tabular}{l} 
5. Radiofármacos \\
\hline 6. Cirugia
\end{tabular} \\
\hline $\begin{array}{l}\text { 7. Otros: inmunoterapia, ácido trans-retinoico, } \\
\text { calcitonina, agentes inhibidores de la angiogénesis } \\
\text { y otros }\end{array}$ \\
\hline
\end{tabular}


todavia en estudio para determinar su eficacia en esta patología, la pauta de tratamiento y las indicaciones.

\section{TERAPIA HORMONAL}

El primer escalón en el tratamiento de pacientes con cáncer de próstata metastásico recién diagnosticados y que, por lo tanto, no han recibido tratamiento previo, está constituido por la ablación androgénica, la cual consigue una tasa de respuestas del $60-80 \%{ }^{7,8}$ a nivel del tumor primario y de las metástasis de partes blandas. Su mecanismo de acción se basa en el hecho de que los andrógenos estimulan la síntesis de DNA y la proliferación celular y su supresión desencadena el fenómeno de apoptosis (muerte celular programada) ${ }^{9}$.

La deprivación androgénica se puede conseguir mediante cuatro mecanismos diferentes ${ }^{10}$ como son la inhibición de la síntesis de andrógenos, el bloqueo periférico de los receptores androgénicos, la ablación de los órganos productores de andrógenos y la interacción con los mecanismos de regulación fisiológica de la producción androgénica. A nivel práctico se traduce en dos posibilidades: la castración quirúrgica o el tratamiento con fármacos hormonales (Tabla II).

\section{TABLA II}

FÁRMACOS PARA LA DEPRIVACIÓN ANDROGÉNICA

1. Estrógenos: dietilestilbestrol

2. Análogos LH-RH: leuprorelina, goserelina, triptorelina, buserelina

3. Antiandrógenos

Esteroideos: acetato de ciproterona, acetato de megestrol

No esteroideos: flutamida, bicalutamida

4. Inhibidores de la esteroideogénesis: ketoconazol y aminoglutetimida

La castración quirúrgica bilateral es una práctica poco habitual, a pesar de que con ella se consigue una deprivación precoz con mejorías sintomáticas en el $72-80 \%$ de los casos, debido a la nula aceptación psicológica por parte de los enfermos y porque tiene el inconveniente de ser irreversible. El empleo de fármacos, sin embargo, se encuentra ampliamente extendido siendo la tendencia actual el bloqueo androgénico combinado (CAB: combined androgen blockade).
Aunque continúa siendo polémico cual es el momento adecuado para iniciar el tratamiento hormonal, y algunos autores defienden esperar hasta la aparición de los sintomas ${ }^{11}$, estudios llevados a cabo por el NCI Intergroup ${ }^{12}$ y por la EORTC $^{13}$ demuestran que en pacientes con buen estado general y volúmenes tumorales pequeños se alarga el tiempo hasta la progresión y se prolonga la supervivencia cuando el inicio del tratamiento es precoz.

En pacientes que presentan mal pronóstico en el momento del diagnóstico (enfermedad ósea extensa y status performance bajo); en enfermos que, siendo inicialmente de buen pronóstico, no normalizan las cifras de PSA en un periodo de 32 semanas de tratamiento con bloqueo hormonal y en aquellos que desarrollan enfermedad andrógeno-independiente ( 2 años desde el inicio de la terapéutica hormonal) es necesario valorar el uso de otras terapias de segunda línea.

\section{Bloqueo androgénico combinado}

El bloqueo androgénico combinado o terapia de asociación se basa en la utilización de combinaciones de fármacos o de orquiectomía quirúrgica con un medicamento hormonal y su meta es lograr mejorar los resultados de la terapia estándar. La combinación más habitual es la de un análogo de LH-RH con un antiandrógeno. La base teórica del bloqueo combinado es la existencia de grupos de células que no responden al bloqueo hormonal o que son sensibles a la acción de dosis bajas de dehidrotestosterona. Bloqueando la acción de dichas hormonas androgénicas sintetizadas en la glándula suprarrenal y que, por tanto, no responden a la inhibición de los análogos de LH-RH que se produce a nivel central, se logrará el bloqueo hormonal completo.

Los estudios dirigidos a demostrar la superioridad del bloqueo androgénico frente a la monoterapia han sido numerosos ${ }^{14,15}$. La terapia combinada se ha mostrado más eficaz que la orquiectomía quirúrgica en pacientes con enfermedad esquelética mínima y/o metástasis de partes blandas. Sin embargo, la supresión androgénica, tan eficaz en disminuir las masas tumorales de partes blandas, no tiene capacidad para eliminar la enfermedad ósea. Esto hace que los pacientes con afectación ósea extensa (más de seis localiza- 
ciones), lesiones predominantemente periféricas y status performance bajo no se beneficien con el bloqueo androgénico combinado.

Los criterios para una combinación ideal incluyen supresión rápida del andrógeno, facilidad de reversibilidad, ausencia de reacción de llamarada, baja toxicidad, efecto dosis-respuesta y facilidad de administración. Los esquemas recomendados varían de unos autores a otros. Goldenberg ${ }^{9}$ aconseja durante las cuatro semanas iniciales de tratamiento administrar antiandrógenos esteroideos o no esteroideos, solos o combinados con estrógenos a dosis bajas, con lo que se consigue disminuir los niveles de testosterona hasta niveles de castración en 3-7 días y se previene el fenómeno de llamarada. Pasado este periodo de tiempo se inician los análogos de LH-RH a la vez que se suprime la administración del estrógeno, pero el antiandrógeno se mantiene para evitar los episodios de sofocos. Este esquema tiene las ventajas de un rápido inicio de acción; baja incidencia de sofocos; ser reversible y bien tolerado por los pacientes. Su principal desventaja es la disminución de la libido y de la potencia sexual.

\section{Enfermedad andrógeno-independiente}

La eficacia del tratamiento ablativo en pacientes metastásicos de buen pronóstico es del 60$80 \%$, pero este efecto es finito y la mayoría de los enfermos desarrollan una resistencia al tratamiento hormonal (estadio D3) en un periodo de dos años ${ }^{16,17}$. La supervivencia media en esta fase, en la que el tumor escapa al control hormonal, es de menos de un año. La actitud a seguir en todos los pacientes es la retirada del antiandrógeno, que produce una mejoría bioquímica, clínica y radiográfica. Los análogos de LH-RH, sin embargo, deben mantenerse pues siempre existe una proporción de células tumorales sensibles a la testosterona ${ }^{18}$. Transcurridas seis semanas desde la retirada del antiandrógeno, y dependiendo de la respuesta alcanzada, se evaluará la conveniencia de introducir un tratamiento de segunda línea, considerando siempre el hecho de que cualquier otro régimen terapéutico tendrá un efecto temporal y que ninguna de las terapias de las que se disponen actualmente produce remisiones duraderas aunque algunas han demostrado un claro beneficio paliativo.

\section{Bloqueo androgénico intermitente}

En un intento por suprimir o retrasar la aparición de la condición andrógeno-independiente, se estableció la hipótesis de que las células malignas que sobrevivian a la supresión androgénica podían ser conducidas hacia una vía de diferenciación normal mediante la restauración de los niveles habituales de andrógenos, preparándolas así para sufrir la apoptosis tras una nueva supresión androgénica ${ }^{19}$. De esta manera, la apoptosis se produciría múltiples veces con ciclos sucesivos de supresión androgénica.

En los estudios clínicos realizados la pauta habitual para realizar el bloqueo intermitente es interrumpir la supresión androgénica tras 36 semanas de tratamiento, siempre y cuando las cifras de PSA se hayan reducido por debajo de los $4 \mathrm{ng} / \mathrm{ml}$. La testosterona sérica retorna a sus cifras normales en un periodo que varía entre $1 \mathrm{y}$ 26 semanas tras la interrupción del tratamiento y esto da lugar a la recuperación de la libido y de la potencia sexual hasta unos niveles iguales o muy similares a los existentes antes del inicio de la terapia hormonal. El nuevo ciclo de supresión androgénica se realizará cuando el PSA alcance cifras de $20 \mathrm{ng} / \mathrm{ml}$. Los ciclos se repiten de esta manera hasta que el tumor se convierte en resistente al tratamiento hormonal. En la revisión realizada por Rambeaud ${ }^{20}$ se concluye que el bloqueo intermitente retrasa la aparición de la hormono-resistencia aunque no la previene. La calidad de vida del paciente mejora gracias a los periodos de función sexual normal. Resta ahora por establecer mediante más estudios las indicaciones precisas de este tratamiento y clarificar si influye de alguna manera en la supervivencia.

\section{Inhibidores de la esteroideogénesis}

Incluidos en el grupo de terapia hormonal tenemos los fármacos inhibidores de la sintesis de andrógenos adrenales: ketoconazol y aminoglutetimida. El primero provoca una castración química en menos de 24 horas con dosis de 1200 mg (seis veces la dosis a la que se emplea como antifúngico) pero su efecto revierte rápidamente al suspender el tratamiento y su hepatotoxicidad limita el uso a largo plazo ${ }^{21}$. Por esto, su principal indicación está en enfermos con compresión medular o coagulopatía. La aminoglutetimida 
tiene efectos similares al ketoconazol y debe administrarse conjuntamente con hidrocortisona ${ }^{22,23}$. Ambos son medicamentos de segunda línea. Dentro de este apartado podemos incluir asî mismo la terapia con corticoides a dosis bajas. Este es un tratamiento barato, bien tolerado y de carácter estrictamente paliativo.

\section{RADIOTERAPIA EXTERNA}

La radioterapia externa tiene un papel fundamental en el tratamiento sintomático de las metástasis óseas. Está indicada en el dolor secundario a lesiones tumorales, cuando existe riesgo de fractura o aplastamiento por destrucción de más del $50 \%$ de la cortical, principalmente en huesos de carga, y en compresiones de médula o de raíces nerviosas. Dependiendo del número de lesiones sintomáticas el tratamiento radioterápico puede ser unifocal, multifocal o hemicorporal.

\section{Radioterapia externa uni o multifocal}

En los casos en los que existe riesgo de fractura, compresión de tejido nervioso o dolor localizado secundario a metástasis se emplean campos de irradiación centrados en el lugar de la lesión que se quiere paliar.

La radioterapia con intención antiálgica consigue resultados positivos, es decir, control completo del dolor o reducción en la dosis de analgésicos, en más del $70-75 \%{ }^{24,25}$ de los pacientes tanto con esquemas hipofraccionados como con dosis únicas. Un estudio randomizado multicéntrico reciente ${ }^{26}$ ha demostrado en pacientes metastásicos con diferentes orígenes tumorales, de los cuales el 34\% eran enfermos con cáncer de próstata, que dosis únicas de 8 Gy logran un alivio del dolor igual al conseguido con 4 fracciones de 500 cGy, sin diferencias significativas en la duración de la respuesta, el número de nuevas localizaciones sintomáticas ni en la necesidad de reirradiación. Por tanto, una dosis única de 800 cGy es una buena opción de tratamiento siempre y cuando no se trate de una irradiación multifocal extensa.

Las lesiones con riesgo de producir una fractura ósea se tratan mediante radioterapia externa y fijación interna o externa, según las condiciones del paciente, para prevenir la rotura y si ésta ya está establecida el tratamiento consistirá en la estabilización quirúrgica seguida de radioterapia, dependiendo de la esperanza de vida del paciente, con o sin terapia sistémica.

La compresión nerviosa, secundaria al crecimiento de las metástasis del cuerpo vertebral dentro del canal medular o por extensión a través del foramen de tumores paravertebrales, es relativamente frecuente en los pacientes con cáncer de próstata ya que el lugar más frecuente de metastatización es vertebral, especialmente la región lumbosacra a través del plexo venoso de Batson $^{27}$. La compresión medular es una urgencia que debe ser tratada de forma inmediata, bien mediante la descompresión quirúrgica bien con radioterapia, en función de la supervivencia estimada del paciente y del tiempo de instauración del cuadro neurológico. La cirugía puede oscilar desde la laminectomía hasta la cimentación con metil-metacrilato, siempre con el objetivo de descomprimir la médula espinal y estabilizar el raquis. El tratamiento con radioterapia se realiza mediante un campo posterior directo o mediante dos campos laterales y opuestos, dependiendo del nivel de la compresión, que incluyan toda la extensión de médula afecta más un cuerpo vertebral superior y otro inferior, dosificados a plano medio en el caso de dos campos o a la profundidad de la médula medida con TAC o RNM en el caso de un campo único. El fraccionamiento empleado con más frecuencia es el de 5 fracciones de 400 cGy/día. El tratamiento radioterápico debe ser complementado siempre con la administración endovenosa de dexametasona a dosis de $20 \mathrm{mg}$ iniciales seguidos de $4 \mathrm{mg} / 6$ horas.

\section{Irradiación hemicorporal}

En la enfermedad metastásica con múltiples localizaciones sintomáticas resulta más adecuado realizar una irradiación hemicorporal que un tratamiento multifocal que puede llegar a ser impracticable. La irradiación hemicorporal logra no sólo un adecuado control del dolor sino que es capaz de retrasar la aparición de síntomas en lesiones ya existentes así como el desarrollo de nuevas lesiones ${ }^{28,29}$.

La irradiación subtotal se divide en superior, media e inferior, situándose habitualmente la separación entre superior e inferior en una línea 
que atraviesa el disco entre L3-L4, aunque los campos pueden acomodarse a las peculiaridades de cada paciente. El tratamiento se realiza mediante dos campos AP-PA dosificados a la mitad del espesor del paciente y pueden conformarse para proteger áreas previamente irradiadas o tejido sano que no interese irradiar. Cuando esté indicado el tratamiento de todo el cuerpo, la irradiación de la mitad superior e inferior debe separarse por un periodo mínimo de 68 semanas para permitir la recuperación de la médula ósea ${ }^{30}$.

Referente a la dosis y al fraccionamiento se ha comprobado $^{25,31}$ que la administración de una dosis única, que varía entre 6 Gy en mitad superior y 8-10 Gy en mitad inferior, consigue una mejoría rápida del dolor en 48 horas en un porcentaje no muy elevado de pacientes $(20 \%)$, con escasos efectos secundarios. La irradiación hemicorporal fraccionada, con dosis que oscilan entre 17,5 Gy en 7 fracciones y 30 Gy en 10 sesiones, tiene una respuesta más tardía (1-2 semanas) con mayores efectos secundarios pero, sin embargo, la tasa de respuestas alcanza el $80 \%$ y la duración del efecto paliativo es más prolongada. La supervivencia no difiere de un grupo a otro. En vista a estos resultados, parece lógico emplear dosis única en pacientes con corta expectativa de vida que requieren una paliación inmediata y esquema fraccionado en el resto.

Los efectos secundarios agudos incluyen toxicidad gastrointestinal, que puede evitarse premedicando a los pacientes con antieméticos, corticoides y prehidratación, e hipoplasia medular, la cual afecta a la mayoría de los pacientes al cabo de unas semanas tras la irradiación y en ocasiones requiere soporte transfusional. La toxicidad más grave es la neumonitis intersticial y debe prevenirse limitando la dosis pulmonar a $6 \mathrm{~Gy}^{29}$.

En nuestro servicio del Clínico de Valencia, entre 1996 y 2000, se han tratado 43 pacientes diagnosticados de adenocarcinoma de próstata con metástasis óseas. El rango de edad está entre 57 y 81 años y la media es de 72 años. Todos ellos presentaban sintomatología derivada de las lesiones óseas, bien dolor, bien complicaciones neurológicas por compresión medular, radicular o de la cola de caballo. El 50\% de los enfermos requirió la irradiación de varias localizaciones de forma simultánea o secuencial, con un rango de entre 7 y 2 localizaciones, y en dos ocasiones se volvió a irradiar la misma zona por recurrencia del dolor tras una buena respuesta inicial. La columna fue la zona de irradiación más frecuente $(39 \%$ de las irradiaciones) pero si diferenciamos entre columna cervical, dorsal y lumbar es la cadera (cabeza femoral, acetábulo, rama isquiopubiana) la región más frecuentemente irradiada (30\%). Las áreas menos frecuentes fueron un metatarsiano y la rodilla, ambas irradiadas en un mismo paciente.

El tratamiento consistió en todos los casos en telecobaltoterapia local con intención paliativa. $\mathrm{El}$ fraccionamiento más empleado fue cinco fracciones de 400 cGy/día, que se administró en el $53 \%$ de las irradiaciones, seguido de cuatro fracciones de 500 cGy/día (17\%) y diez fracciones de 300 cGy/día (7\%). En el resto de las localizaciones se emplearon fracciones de 500 cGy semanales en número variable según la respuesta clínica. En ningún paciente se administraron fracciones únicas de 800 cGy. En el caso del fraccionamiento más usado (5 x 400 cGy/día) la respuesta inmediata más habitual fue parcial (39\%) con disminución de los requerimientos de medicación analgésica, total en un $9 \%$ y progresión o estabilidad de la clínica en otro 9\% en ambas posibilidades. En un 33\% de los casos no se valoró la respuesta a la irradiación.

En los casos de síndrome de compresión medular $(7 \%$ del total de irradiaciones) sólo un paciente presentó respuesta completa que persistía a los dos meses del tratamiento.

\section{QUIMIOTERAPIA}

El tratamiento con agentes citotóxicos no ha logrado prolongar la media de supervivencia de diez meses que presentan estos pacientes tras el desarrollo de la enfermedad hormono-independiente. En consecuencia, la identificación de agentes que por sí solos o en combinación sean efectivos frente a las células tumorales que escapan al control hormonal, constituye una necesidad perentoria en esta patología.

En este punto es importante reseñar que algunos casos considerados como cáncer de próstata andrógeno-independiente son tumores anaplásicos de célula pequeña, puros ( $1 / 3$ de los casos) o 
mixtos por la presencia de un porcentaje de células de adenocarcinoma (2/3 de los casos). Estos tumores se caracterizan por segregar neuropéptidos activos, motivo por el cual se suelen presentar clínicamente acompañados de síndromes paraneoplásicos. Estos tumores son muy sensibles a las combinaciones de quimioterapia estándar en el tratamiento del carcinoma microcítico pulmonar (CAV, platino + etopósido), por lo que estos esquemas constituyen su tratamiento de elección. Estos pacientes no deben ser tratados siguiendo las pautas del adenocarcinoma de próstata ya que son resistentes a dichas terapias.

Se están desarrollando actualmente nuevos fármacos que actúan de forma selectiva sobre algunos blancos tumorales. Dichos fármacos están siendo objeto de diferentes investigaciones y estudios en un intento de encontrar un tratamiento quimioterápico útil y con pocos efectos secundarios en estos pacientes con cáncer de próstata en estadio avanzado resistentes a la terapia hormonal.

Inhibidores de la formación de los microtúbulos

$\mathrm{El}$ fosfato de estramustina es una conjugación de mostaza nitrogenada con estradiol que presenta acción mixta, hormonal y no hormonal. Actúa alterando la función de los microtúbulos mediante la inhibición de la unión de la tubulina y de las proteínas asociadas a los microtúbulos ${ }^{32-}$ 34. La estramustina usada como monoterapia en pacientes con enfermedad hormono-independiente ha demostrado en un estudio fase II, en el que se empleó como criterio de respuesta el PSA, una tasa de respuestas del 14\% en 44 pacientes a los que se trató con estramustina oral a dosis de $15 \mathrm{mg} / \mathrm{kg} / \mathrm{dí}^{35}$. Ante estos pobres resultados se estableció la hipótesis de que la asociación a la estramustina de otros fármacos que también actúen alterando los microtúbulos podría mejorar los resultados. Los alcaloides de la vinca, vinblastina y vincristina, producen aglutinación de la tubulina, alterando así la formación de los microtúbulos. Dado que en los experimentos "in vitro" se ha observado que el mayor efecto sinérgico lo presenta la combinación de estramustina y vinblastina ${ }^{36}$, es esta asociación la que se ha valorado en varios estudios no randomizados y en un ensayo fase III randomizado conducido por el
Hoosier Oncology Group y la Fox Chase ${ }^{37}$, en los que se trataron pacientes con enfermedad hormono-independiente.

Aunque los resultados de este estudio no demuestran un claro beneficio en términos de supervivencia global de la combinación de vinblastina y estramustina frente a la monoterapia sí se objetiva una mejoría en el control del dolor, la reducción de las masas medibles y la disminución de las cifras de PSA.

Los fármacos de la familia de los taxanos poseen la capacidad de actuar directamente estabilizando la función de los microtúbulos, inhibiendo de esa manera la progresión mitótica e induciendo la apoptosis. Resultados preliminares de estudios fase I y fase $\mathrm{II}^{38-40}$, en los que se combinan derivados de taxanos (docetaxel y paclitaxel) con estramustina, apuntan a que los resultados pueden ser superiores a los obtenidos con la combinación de vinblastina. De todo ello se deduce que, son necesarios más estudios randomizados con combinaciones de drogas inhibidoras de la formación de los microtúbulos para obtener tratamientos más efectivos frente al cáncer de próstata avanzado, en situación de resistencia a las terapias hormonales.

Fármacos con actividad sobre la matriz nuclear

La matriz nuclear se altera en la transformación celular a la malignidad dando lugar a trastornos en la replicación del DNA y en la expresión de los genes. La estramustina y el etopósido en monoterapia tienen un efecto modesto sobre el crecimiento celular, pero combinados presentan un efecto sinérgico sobre la matriz nuclear. Basado en este hecho se diseñó un ensayo fase $\mathrm{II}^{41}$ con estramustina y etopósido vía oral combinados con paclitaxel endovenoso. Los pacientes incluidos en el estudio eran enfermos con cáncer de próstata avanzado hormono-independiente, quienes habían recibido previamente terapia hormonal mediante deprivación androgénica. Los resultados muestran una disminución del PSA mayor del 50\% en un $52 \%$ de los casos y $52 \%$ de respuestas objetivas (parciales y completas) en la enfermedad medible.

Inhibidores de los factores de crecimiento

La suramina es una naftilurea polisulfato que tiene actividad frente a los factores de crecimiento, 
inhibiendo la unión de éstos con sus receptores. También actúa inhibiendo la degradación de los glicosaminglicanos, la proteinquinasa $\mathrm{C}$, la glucólisis y la movilidad celular.

Estudios preclínicos demuestran que este fármaco puede tener actividad frente al cáncer de próstata, objetivándose en un modelo animal con líneas celulares de cáncer de próstata hormonoresistente $^{42}$ que la suramina tiene un efecto significativo sobre las metástasis hepáticas pero no sobre las óseas, donde el flujo sanguíneo es menor. Sin embargo, la respuesta antitumoral sólo se logra cuando se mantienen unos niveles en plasma superiores a 250 microgramos/mililitro, lo que constituye un importante problema si consideramos que la toxicidad limitante de dosis, principalmente la neurológica, se encuentra directamente relacionada con la duración de la exposición al fármaco y con niveles plasmáticos mantenidos de 300 microgramos/mililitro o más. Por ello, se pensó que la combinación de la suramina con otros agentes permitiría disminuir las dosis de suramina, con la consiguiente reducción de la toxicidad, a la vez que se mantiene su efecto antitumoral.

Los resultados obtenidos son irregulares. En un estudio fase $\mathrm{II}^{43}$ en el que se combinó suramina con epidoxorrubicina la toxicidad fue intolerable y las respuestas objetivas nulas. En otro estudio fase II ${ }^{44}$ con pacientes en estadio D2 y D1 con mal pronóstico y que no habían recibido tratamiento previo, se combinó suramina con terapia hormonal, manteniéndose las concentraciones de suramina entre 175 y 300 microgramos/mililitro. Los resultados son optimistas, con una alta tasa de respuestas objetivas y una toxicidad moderada y reversible. Sin embargo, un estudio posterior, conducido por el SOG ${ }^{45}$, con enfermos de similares características (enfermedad extendida, no tratamientos previos), quienes recibieron tratamiento combinado con suramina y bloqueo hormonal, concluye que la suramina tiene un interés limitado en esta patología y una toxicidad elevada.

\section{BISFOSFONATOS}

Los bisfosfonatos son análogos sintéticos del pirofosfato que actúan como potentes reguladores del recambio óseo. Su mecanismo de acción en procesos tumorales, que ha sido demostrado con células de carcinoma de mama y de próstata $^{46}$, se basa en la inhibición de la adhesión de las células cancerígenas a la matriz osteoblástica extracelular de una forma dosis dependiente y sin afectar a la unión de los fibroblastos a la misma. Por ello, disminuyen la reabsorción ósea sin afectar a la mineralización del hueso.

Consecuencia de esta inhibición es una reducción de los hechos asociados con las metástasis óseas como el dolor, las fracturas y la hipercalcemia, así como una reducción de la carga tumoral en el hueso y, en experimentos animales, una supervivencia alargada.

Numerosos estudios clínicos ${ }^{47-49}$ con clodronato y pamidronato han demostrado su eficacia en el tratamiento de enfermos con metástasis óseas osteolíticas por cáncer de mama o mieloma múltiple, obteniéndose resultados favorables en el control del dolor y en la mejoría de la calidad de vida. Otras conclusiones a las que se llega en estos estudios son que la recalcificación de las lesiones líticas ocurre en el $25 \%$ de los pacientes; en más del 95\% se logra una disminución de la reabsorción ósea medida mediante la excreción de hidroxiapatita en la orina, aunque este hecho choca con la falta de respuesta clínica en todos los enfermos; los fármacos son bien tolerados, objetivándose sólo una hipocalcemia asintomática en algunos pacientes, la cual revierte espontáneamente en 3-4 semanas y el retratamiento es eficaz únicamente en los enfermos que respondieron inicialmente.

Sin embargo, la utilidad clínica de los bisfosfonatos en metástasis de origen prostático no está tan bien definida como en las patologías antes mencionadas. Estudios en ratas con cáncer de próstata ${ }^{50-53}$ mostraron que los bisfosfonatos inhiben la osteolisis inducida por el tumor aunque no actúan sobre el proceso osteoblástico. Estudios preliminares con enfermos con cáncer de próstata metastásico, los cuales fueron tratados con pamidronato ${ }^{54}$ y clodronato ${ }^{55}$, mostraron la capacidad de estos medicamentos para producir alivio del dolor incluso en aquellos pacientes en los que predominaba el componente esclerótico. Esto es debido a que siempre existe un fenómeno lítico importante en estas lesiones aunque el carácter predominante sea el blástico. En estos estudios no se hicieron mediciones de PSA. 
Existen ya varios bisfosfonatos disponibles en el mercado. En orden de potencia inhibidora, de mayor a menor, se incluyen ibandronato, risedronato, pamidronato y clodronato. Otros de estos fármacos son etidronato, alendronato y tiludronato.

\section{Pamidronato}

Se han realizado estudios con este fármaco en enfermos metastásicos con cáncer de próstata pero resulta dificultoso evaluarlos conjuntamente dadas las diferentes pautas de administración y las combinaciones que algunos autores realizan con otras modalidades terapéuticas. El pamidronato, que sólo puede administrarse de forma endovenosa, ha sido empleado como bolo único de $120 \mathrm{mg}^{56}$ y en dosis de $30 \mathrm{mg} / 2$ semanas; 60 $\mathrm{mg} / 2$ semanas; $60 \mathrm{mg} / 4$ semanas y $90 \mathrm{mg} / 4$ semanas durante 3 meses $^{57,58}$. Los resultados muestran que no existe una relación dosis-respuesta y que aunque se observaron diferencias estadísticamente significativas en la valoración del dolor por parte de los pacientes al principio y al final del estudio, no se consiguieron reducciones evidentes en la dosis de analgésicos. El fármaco fue bien tolerado.

\section{Clodronato}

El clodronato se comercializa tanto para su uso oral como endovenoso, por lo que la tendencia es a administrar dosis iniciales endovenosas de $300 \mathrm{mg} /$ día, durante un periodo de 6 a 10 días según los autores, seguidas de clodronato oral en dosis de 1600 ó 3200 mg/día ${ }^{59-61}$. Los resultados muestran alivio del dolor de forma parcial o completa en un $37-71 \%$ de los pacientes, con una duración media de la respuesta de 7 semanas y respuesta mantenida de forma prolongada en algunos pacientes que continuaron con el tratamiento oral.

\section{Risedronato}

El risedronato es un bisfosfonato de tercera generación que requiere dosis más bajas que los anteriores pero que no se ha estudiado aún en experiencias clínicas con pacientes cancerosos. Existe un experimento ${ }^{62}$ con un modelo de ratones inoculados con células de cáncer de mama en el que se concluye que el tratamiento con risedronato retrasa la progresión de las lesiones exis- tentes a la vez que inhibe la aparición de nuevas localizaciones y disminuye tanto la carga tumoral como el número de osteoblastos en los lugares metastásicos. Sin embargo, no afecta a las lesiones de tejidos blandos adyacentes al hueso.

En una revisión realizada por Clarke ${ }^{1}$ se concluye que los bisfosfonatos pueden emplearse en el tratamiento de las metástasis óseas secundarias a cáncer de próstata ya que disminuyen la osteolisis y mejoran la sintomatología, aunque se desconoce la duración de este efecto, a la vez que son bien tolerados. Sin embargo, son necesarios estudios randomizados para establecer las dosis adecuadas así como la duración del tratamiento, ya que no se pueden extrapolar los resultados de los estudios animales o clínicos realizados con cáncer de mama al cáncer de próstata.

\section{RADIOFÁRMACOS}

En pacientes con lesiones metastásicas múltiples los radioisótopos con afinidad por el hueso constituyen una opción terapéutica que permite el tratamiento simultáneo de varias localizaciones metastásicas óseas de una manera efectiva y con una relación coste-efectividad apropiada. Su toxicidad, además, es reducida ya que presenta una absorción limitada en los tejidos sanos. En EE.UU y Europa están disponibles para su uso en la clínica el estroncio-89 ( $\mathrm{Sr}^{89}$ o metastrón) y el samario-153 ( $\mathrm{Sm}^{153}$ o lexidronam). Ambos han demostrado en numerosos estudios randomizados y prospectivos $^{63,64}$ que reducen de forma significativa el dolor y los requerimientos de analgésicos, que mejoran la calidad de vida y disminuyen el número de tratamientos con radioterapia externa, enlenteciendo la progresión de las lesiones metastásicas dolorosas. El retratamiento es posible, eficaz y con pocos efectos secundarios. Por otra parte, algunos autores apuntan la posibilidad de administrar estos fármacos de manera concomitante con la radioterapia externa para el tratamiento, no sólo de las lesiones sintomáticas sino también de las metástasis ocultas asintomáticas.

Existen otros radiofármacos, aún no disponibles para su uso habitual, que están siendo objeto de estudio en ensayos fase II y III para evaluar su eficacia y compararlos con los mencionados anteriormente. Son el renio-186 $\left(\mathrm{Re}^{186}\right)$ y el DTPA (Tin-117m dietilenotriaminopenta ácido acético). 


\section{Estroncio}

$\mathrm{El}$ estroncio $\left(\mathrm{Sr}^{89}\right)$ es un isótopo radiactivo de la familia del calcio, con una acción similar a éste, que actúa fijándose en los lugares de producción del hueso y que, por lo tanto, se deposita en las metástasis blásticas. Se trata de un emisor $\beta$ casi puro por lo cual su acción alcanza a muy pocos milímetros alrededor del lugar donde se deposita. Su principal inconveniente es que no tiene acción sobre las metástasis líticas, por lo que su utilidad está muy limitada en la mayoría de los procesos neoplásicos, no así en el cáncer de próstata en el que predominan las metástasis de carácter blástico. Los estudios publicados ${ }^{65}$ demuestran una paliación eficaz del dolor hasta en el $75 \%$ de los casos tras administrar de 1,11 a $1,48 \mathrm{mBq} / \mathrm{kg}$ del fármaco, así como efectos superiores en los pacientes tratados con estroncio-89 frente a placebo $^{66}$ y a estroncio frío ${ }^{67}$. $\mathrm{El} \mathrm{Sr}{ }^{89}$ puede administrarse nuevamente si existe reaparición del dolor tras un mínimo de doce semanas del primer tratamiento. Su principal toxicidad limitante de dosis es la plaquetopenia, aunque suele recuperarse de forma completa pasadas unas semanas de la administración. No existen datos publicados sobre su efecto sobre los niveles de PSA.

\section{Samario}

El Sm-153-EDTMP (samarium ${ }^{153}$ etileno diamina tetrametileno fosfonato) es un bisfosfonato marcado que se caracteriza por una emisión gamma en pequeña proporción y $\beta$ en su mayor parte, la cual es la responsable del efecto terapéutico. El bisfosfonato es el causante de la fijación del fármaco en el hueso. Su vida media es de 1,9 días. En todos los estudios publicados ${ }^{68-70} \mathrm{se}$ consiguen mejorias del dolor en pacientes con metástasis óseas secundarias a diferentes tumores primarios hasta en un $84 \%$ de los casos. La principal toxicidad observada es la neutropenia y la plaquetopenia, reversibles en todos los casos. La dosis empleada es una dosis única de 0,5 $\mathrm{mCi} / \mathrm{Kg}(18,5 \mathrm{mBq} / \mathrm{Kg})$ o de $1,0 \mathrm{mCi} / \mathrm{Kg}(37$ $\mathrm{mBq} / \mathrm{Kg}$ ) y es aquí donde se produce la discrepancia en los resultados ya que en el estudio de la Universidad de Miami ${ }^{69}$ obtienen el mayor porcentaje de respuestas en el grupo al que se administró $1,0 \mathrm{mCi} / \mathrm{Kg}$, mientras que en el estudio chino $^{70}$ la respuesta y los efectos secundarios fueron ambos dosis-independientes. Por tanto, son necesarios más estudios para determinar la dosis adecuada.

\section{Renio}

El Re-86-HEDP (rhenium ${ }^{186}$ [Tin] hidroxietilideno difosfonato) es, al igual que el samario, un bisfosfonato marcado que emite radiación $\beta \mathrm{y}$ radiación $\gamma$. Ésta última les confiere ventaja sobre el estroncio ya que facilita los cálculos dosimétricos.

Estudios fase $\mathrm{II}^{71,72}$ en pacientes con cáncer de próstata andrógeno-independiente a los que se administró renio, en dosis que variaron entre 1251 y 4336 mBq (33,8-117,2 mCi), demostraron que la radiación al cuerpo entero, a la vejiga y al riñón era bien tolerada. No obstante, se provocó neutropenia y plaquetopenia grado III en los grupos que recibieron dosis más elevadas y, en algunos casos, estas alteraciones fueron irreversibles. Otros efectos secundarios son neuropatía periférica y un efecto "llamarada" con incremento del dolor a los 3-6 días de la administración. Se han publicado algunos casos ${ }^{73}$ de neuropatía cerebral transitoria en enfermos con cáncer de próstata tratados con renio. En el estudio del $\mathrm{MSKCC}^{72}$ se evaluó también la repercusión sobre los niveles de PSA, objetivándose disminución del 50\% o más de aquellos en sólo tres de los 27 pacientes, respuesta que no se mantuvo. A pesar de todo, el renio se muestra como un fármaco prometedor en el tratamiento de las metástasis dolorosas del cáncer de próstata, con un efecto analgésico más prolongado que el estroncio. Son necesarios estudios fase III para determinar la dosis y las indicaciones de este radiofármaco.

Actualmente, se están planteando diversas estrategias para aumentar la eficacia de estos fármacos mediante la administración de dosis más altas, radiosensibilizantes que inhiban la reparación del daño subletal y combinaciones con quimioterápicos.

\section{CIRUGíA}

El papel de la cirugía en estos pacientes está limitado a la estabilización de fracturas y descompresión de tejidos nerviosos y, en general, las indicaciones son similares a las de las metástasis de otros orígenes. 
La estabilización quirúrgica de fracturas metafisarias se puede realizar mediante placas y tornillos pero, con este método, como no se estabiliza el hueso entero, existe el riesgo de sufrir nuevas fracturas en otras localizaciones. Una segunda opción es la colocación de prótesis, aunque en estas localizaciones puede ser imposible este procedimiento dada la pérdida de sustancia. Además, es muy difícil valorar la alineación y la longitud del hueso, por lo que suelen producirse asimetrías e inestabilidad de la articulación.

Las fracturas diafisarias se tratan con éxito mediante fijación intramedular, siendo además relativamente sencillo rellenar las áreas donde se ha perdido sustancia con cementos de metacrilato. El resultado en términos de alineación y longitud del miembro así como funcional es bueno.

La intervención quirúrgica en los casos de compresión medular o nerviosa puede ser de primera elección en pacientes con buen estado general y una expectativa de vida prolongada. En el resto de pacientes debe considerarse en tumores que se han demostrado radiorresistentes; en aquellos que ya han recibido radioterapia a dosis plenas en el campo afecto y cuando exista inestabilidad vertebral ${ }^{74,75}$.

En todos los casos debe valorarse cuidadosamente el potencial beneficio de la intervención, la duración prevista de la hospitalización y convalecencia y, por encima de todo, la esperanza de vida del paciente.

\section{NUEVAS ESTRATEGIAS}

El mejor conocimiento que se tiene en la actualidad de la biología de los tumores, incluyendo la apoptosis, la diferenciación, el ciclo celular y la angiogénesis, ha proporcionado numerosos nuevos blancos para la terapia antitumoral. Los fármacos que modulen estos procesos pueden originar efectos citotóxicos o citostáticos. El principal problema es que estas terapias van dirigidas contra un único blanco celular, por lo que los parámetros empleados clásicamente para la evaluación de nuevos fármacos pueden no ser útiles para determinar el beneficio clínico de las nuevas drogas.

\section{Inhibidores de la angiogénesis}

Siguiendo a la transformación maligna, las células tumorales y el estroma adyacente segregan factores de crecimiento y enzimas proteolíticas que inducen la formación de nuevos vasos (angiogénesis) y la degradación de la matriz, facilitando de esta manera el desarrollo de la neoplasia. Dos familias de enzimas proteolíticas están implicadas en este proceso: metaloproteasas (MMPs) y uroquinasas (uPA $)^{76}$. Las células del cáncer de próstata segregan grandes cantidades de MMPs y niveles bajos de inhibidores endógenos de metaloproteasas (TIMPs), ocasionando un balance excesivo de MMPs. Además, las líneas celulares de cáncer de próstata que expresan los niveles más elevados de MMPs metastatizan con mayor frecuencia, principalmente al hueso y al pulmón. Por ello, las terapias que bloqueen la acción de MMPs y uPA ocasionarían una marcada reducción en el crecimiento del tumor, convirtiendo a estas enzimas en blancos de la terapia antitumoral.

Estudios in vitro con líneas celulares de cáncer de próstata demuestran que sustancias como la bombesina ${ }^{77}$, la interleuquina- $10^{78}$, doxiciclina $\mathrm{y}$ tetraciclinas modificadas químicamente ${ }^{79}$ pueden actuar regulando la angiogénesis. Los estudios in vivo con ratas ${ }^{79}$ muestran que tanto la doxiciclina como la tetraciclina modificada CMT3 reducen la tasa de metástasis pulmonares, aunque sólo la CMT-3 es capaz de disminuir la tasa de crecimiento tumoral. Ambos fármacos presentan mínima toxicidad sistémica y tienen la gran ventaja de que en humanos se pueden administrar vía oral.

Estos estudios preclínicos proporcionan la base para otros estudios clínicos con pacientes con enfermedad diseminada.

Fármacos que actúan sobre la diferenciación celular

La diferenciación celular constituye otro blanco terapéutico, ya que si se consigue ralentizar la tasa de progresión tumoral un paciente podría permanecer asintomático durante más tiempo, aunque no se haya podido erradicar el tumor.

Los retinoides son unos compuestos que se unen a los receptores nucleares actuando como 
factores de transcripción nuclear. El ácido retinoico ha demostrado in vitro efectos citotóxicos así como sobre la diferenciación celular y sobre la expresión del TGF $\alpha$. En modelos animales inhibe el desarrollo tumoral. Esta actividad ya ha sido ensayada en otros tumores, como los segundos tumores de cabeza y cuello, en los cuales ha demostrado su eficacia ${ }^{80}$. Sin embargo, en un estudio fase $\mathrm{II}^{81}$, con 26 pacientes afectos de cáncer de próstata en situación de hormono-resistencia, demostró una mínima actividad sin ninguna respuesta objetiva y tan solo una disminución del PSA del 50\% o más en 4 pacientes.

Otro grupo de sustancias son los péptidos calciotróficos (calcitonina y hormona paratiroidea) quienes desempeñan un papel fundamental en el crecimiento y desarrollo de la matriz ósea normal. En un estudio in vitro ${ }^{\mathrm{i}}$ con tres líneas celulares de cáncer de próstata se observó que estas hormonas tienen un papel importante en la regulación del crecimiento celular y en la metastatización.

\section{Inmunoterapia}

La inmunoterapia está basada en el incremento de la respuesta inmune del organismo enfermo frente al tumor y ha sido empleada principalmente como alternativa para el tratamiento de cánceres metastásicos refractarios a las terapias convencionales, en particular para el melanoma y el carcinoma renal. Estudios ex vivo con sustancias como interleuquina-2 y el factor estimulante de colonias de macrófagos y granulocitos muestran unos resultados prometedores en modelos animales. Diversas variedades de esta terapia están siendo estudiadas para el cáncer de próstata.

El TAG-72 en un antígeno panadenocarcinoma que se expresa en las células de cáncer de próstata, con mayor frecuencia en los bien diferenciados y menos frecuentemente en los pobremente diferenciados. En el MSKCC ${ }^{83}$ se ha realizado un estudio con pacientes afectos de cáncer de próstata en estadio de hormono-resistencia, quienes han sido tratados con el anticuerpo específico del TAG-72 (CC49) marcado con $\mathrm{I}^{131}$. Aunque el fármaco se fija a todos los lugares metastásicos, tanto óseos como extraóseos, sólo cuatro pacientes de 16 tuvieron mejoría del dolor, no hubo ninguna disminución significativa en las cifras de PSA y la terapia se mostró incapaz, a las dosis en las que fue administrada, de erradicar la enfermedad localizada en la médula ósea. La toxicidad limitante de dosis fue la trombocitopenia. Los autores concluyen que en el caso concreto del CC49 sería necesario repetir las dosis para mejorar los resultados, posibilidad que fue descartada.

El antígeno de membrana próstata específico (PSMA) es un antígeno que se expresa en la superficie de células neoplásicas de próstata, con alta expresión en el 90\% de los tumores metastásico y en el $50 \%$ de los primarios, y que es considerado como un nuevo blanco para la terapia antitumoral. Sinha y colaboradores ${ }^{84}$ establecieron la hipótesis de que un anticuerpo específico frente a este antígeno podría actuar como transportador de agentes quimioterápicos, logrando así inhibir de manera específica el crecimiento e inducir la muerte de las células productoras de PSMA. El experimento se realizó con un inmunoconjugado compuesto por IgG anti-PSA y 5-FU, el cual se inyectó a ratones con líneas celulares subcutáneas de células tumorales prostáticas productoras y no productoras de PSA. El análisis del estudio demostró que el compuesto era altamente específico para provocar efectos citotóxicos en las líneas celulares productoras de PSA, sin efectos no deseados en el resto de los órganos del ratón.

$\mathrm{El}$ receptor del factor de crecimiento epidérmico (EGFR) es una proteína que se expresa en la superficie de las células prostáticas. Por ello, se había considerado otro nuevo blanco de las terapias actuales. Sin embargo, estudios recientes ${ }^{85}$ han mostrado una inexplicable reducción del EGFR en las células prostáticas cancerígenas. Este fenómeno se atribuye a la presencia de una variante del receptor (EGFRvIII) que no está presente en la glándula prostática sana pero cuyos niveles se incrementan progresivamente con la gradual transformación de los tejidos hacia el fenotipo maligno. El papel que este receptor mutante puede tener en la iniciación y progresión del crecimiento prostático maligno ofrece una nueva vía de investigación para la inmunoterapia en esta enfermedad. 


\section{REFERENCIAS}

1. CLARKE NW.: The effects of pamidronate disodium treatment in metastatic prostate cancer. Rev Contemp Pharmacother 1998; 9: 205-212.

2. JACOBS SC, LAWSON RK.: Mitogenic factor in human prostate extracts. Urology 1980; 16: 488-491.

3. CLARKE NW, McCLURE J, GEORGE NJR.: Morphometric evidence for bone resorption and replacement in prostate cancer. $\mathrm{Br} J$ Urol 1991; 68: 74-80.

4. BONICA JJ.: Control of bone cancer pain. In: Garattini F, Ed. Bone resorption, metastasis and diphosphonates. New York: Raven Press, 1985: 139-180.

5. MAHADERIA P, RAMOSWAMY A, GREENWALD E et al.: Hypercalcaemia in prostate cancer. Arch Int Med 1983; 143: 1339-1342.

6. JACOBS SC.: The spread of prostate cancer to bone. Urology 1983; 21: 337-344.

7. SCHMID HP, BITON A.: Therapeutic options in advanced cancer of the prostate. Schweiz Rundsch Med Prax 1997 oct 29; 86 (44): 1734-1739.

8. BUBLEY GJ, BALK SP.: Treatment of androgen-independent prostate cancer. Oncologist 1996; 1 (1 \& 2): 30-35.

9. GOLDENBERG SL, BRUCHOVSKY N.: Hormonal manipulation for metastatic prostate cancer. Androgen withdrawal therapy: new perspectives in the treatment of prostate cancer. In: Principles and practices of genitourinary. Derek Raghavan, Howard I Scheer, Steven A Leibel and Paul H Lange, Eds. Lippincott-Raven Publishers, Philadelphia 1997; 57 (1): 583-591.

10. KIRBY RS.: Recent advances in the medical management of prostate cancer. Br J Clin Pract 1996; 50 (2): 88-93.

11. IVERSEN P, RASMUSSEN F, KLARSKON P et al.: Longterm results of danish prostatic cancer group trial 86: goserelin acetate plus flutamide versus orchiectomy in advanced prostate cancer. Cancer 1993; 72: 3851.

12. CRAWFORD ED, EISENBERGER MA, MCLEOD D et al.: A controlled trial of leuprolide with and without flutamide in prostatic carcinoma. N Eng J Med 1989; 321: 419-424.

13. DENIS L, CARNEIRO L, BONO A et al.: Acetato de goserelina más flutamida frente a la orquiectomía bilateral: ensayo fase III EORTC 3583. Urology 1993; 42 (2): 119-130.

14. DENIS L, MURPHY GP.: Overview of phase III trials on combined androgen treatment in patients with metastatic prostate cancer. Cancer 1993; 72 (suppl 12): 3888.

15. Prostate cancer trialists' collaborative group.: Maximum androgen blockade in advanced prostate cancer: an overview of 22 randomized trials with 3283 deaths in 5710 patients. Lancet 1995; 346: 265.

16. BUBLEY GJ, BALK SP.: Treatment of androgen-independent prostate cancer. Oncologist 1996; 1 (1 \& 2): 30-35.

17. LARA PN Jr, MEYERS FJ.: Treatment options in androgen-independent prostate cancer. Cancer Invest 1999; 17 (2): 137-144.

18. FOURNIER G, VALERI A.: Treatment of metastatic prostate cancer: certainty and doubts. Presse Med 1998 dec 5; 27 (38): 1996-2002.

19. AKAKURA K, BRUCHOVSKY N, GOLDENBERG SL.: Effects of intermittent suppression on androgendependent tumours: apoptosis and serum prostate specific antigen. Cancer 1993; 71: 2782.
20. RAMBEAUD JJ.: Intermittent complete androgen blockade in metastatic prostate cancer. Eur Urol 1999; 35 suppl 1: 32-36.

21. MCCONELL JD.: Physiologic basis of endocrine therapy for prostatic cancer. Urol Clin North An 1991; 18: 1.

22. HAVLIN KA, TRUMP DL.: Aminoglutethimide: theoretical considerations and clinical results in advanced prostate cancer. Cancer 1988; 39: 83.

23. SHAW MA, NICHOLS PJ, SMITH HJ.: Aminoglutethimide and ketoconazole: historical perspectives and future prospects. J Steroid Biochem 1988; 31: 137.

24. JANJAN NA.: Radiation for bone metastases: conventional techniques and the role of systemic radiopharmaceuticals. Cancer 1997 oct 15; 80 (8 suppl): 16281645.

25. TORDIGLIONE M, LURAGHI R, ANTOGNONI P.: Role of palliative and symptomatic radiotherapy in bone metastasis. Radiol Med (Torino) 1999 may; 97 (5): 372-377.

26. NIELSEN OS, BENTZEN SM, SANDBERG E, GADEBERG CC, TIMOTHY.: An randomized trial of single dose versus fractionated palliative radiotherapy of bone metastases. Radiother Oncol 1998 jun; 47 (3): 233-240.

27. GELDOF AA.: Models for cancer skeletal metastases: a reappraisal of batson's plexus. Anticancer Res 1997 may-jun; 17 (3): 1535-1539.

28. HAZRA TA, GIRI S.: Prophylactic pelvic girdle irradiation in the treatment of prostatic carcinoma. Int $J$ Radiat Oncol Biol Phys 1981; 7: 817.

29. POULTER CA, COSMATO D, RUBIN P.: A report of RTOG 8206: a phase III study of whether the addition of single dose hemibody irradiation to standard fractionated local field irradiation is more effective than local field irradiation alone in the treatment of symptomatic osseous metastasis. Int $J$ Radiat Oncol Biol Phys 1992; 23: 207.

30. HSIU-SAN LIN, DRZYMALA RE.: Total body and hemibody irradiation. In: Principles and practice of radiation oncology, $3^{\mathrm{d}}$ ed. Pérez Ca, Brady Lw Eds. Lippincott-Raven Publishers, Philadelphia 1997; 11: 333-342.

31. ZELEFSKY MJ, SCHER HI, FORMAN JD, LINARES LA, CURLEY T, FUKS Z.: Palliative hemiskeletal irradiation for widespread metastatic prostate cancer: a comparison of single dose and fractionated regimens. Int $J$ Radiat Oncol Biol Phys 1989; 17 (6): 1281-1285.

32. DAHLLOF B, BILLSTRON A, CABRAL $\mathrm{F}$ et al.: Estramustine depolymerizes microtubules by binding to tubulin. Cancer Res 1993; 53: 4573-4581.

33. SPEICHER LA, LAING N. BARONE LR et al.: Interaction of an estramustine photoaffinity analogue with cytoskeletal proteins in prostate carcinoma cells. Mol Pharmacol 1994; 46: 866-872.

34. TEW KD, GLUCKER JP, HARTLEY-ASP B et al.: Preclinical and clinical perspectives on the use of estramustine as an antimitotic. Drug Pharmacol Ther 1992; 56: 323-339.

35. YOGADA A, SMITH JA, SOLOWAY MD et al.: Phase II study of estramustine phosphate in advanced hormone-refractory prostate cancer with increasing prostate specific antigen levels. $J$ Urol 1991; 145: 384a (abstr).

36. MAREEL MM, STORME GA, DRAGONETTI CH et al.: Anti-invasive activity of estramustine on malignant mo4 mouse cells and on du-145 human prostate carcinoma cells in vitro. Cancer Res 1988; 48: 1842-1849. 
37. HUDES G, EINHORN L, ROSS E et al.: Vinblastine versus vinblastine plus oral estramustine phosphate for patients with hormone-refractory prostate cancer: a Hoosier Oncology Group and Fox Chase Network phase III trial. J Clin Oncol 1999 oct; (10): 3160-3166.

38. HUDES GR, NATHAN F, KHATER C et al.: Phase II trial of 96-hour paclitaxel plus oral estramustine phosphate in metastatic hormone-refractory prostate cancer. $J$ Clin Oncol 1997; 15: 3156-3163.

39. KREIS W, BUDMAN DR, FETTEN J et al.: Phase I trial of the combination of daily estramustine phosphate and intermittent docetaxel in patients with metastatic hormone-refractory prostate carcinoma. Ann Oncol 1999; 10: 33-38.

40. PETRYLAK DP, MACARTHUR RB, O'CONNOR J et al. Phase I trial of docetaxel with estramustine in androgen-independent prostate cancer. J Clin Oncol 1999; 17: 958-967.

41. SMITH DC, ESPER P, STRAWDERMAN M, REDMAN B, PIENTA KJ.: Phase II trial of oral estramustine, oral etoposide and intravenous paclitaxel in hormonerefractory prostate cancer. J Clin Oncol 1999 jun; 17 (6): 1664-1671.

42. KOBASYASHI T, KOSHIDA K, ENDO Y et al.: A chick embryo model for metastatic human prostate cancer. Eur Urol 1998 aug; 34 (2): 154-160.

43. MIGLIETTA L, CANNOBIO L, GRANETTO C, VANNOZZI MO, ESPOSITO M, BOCCARDO F.: Suramin/epidoxorubicin association in hormone-refractory prostate cancer: preliminary results of a pilot phase II study. $J$ Cancer Res Clin Oncol 1997; 123 (7): 407-410.

44. DAWSON NA, FIGG WD, COOPER MR et al.: Phase II trial of suramin, leuprolide and flutamide in previously untreated metastatic prostate cancer. $J$ Clin Oncol 1997 apr; 15 (4): 1470-1477.

45. HUSSAIN M, FISHER EI, PETRYLAK DP et al.: Androgen deprivation and four courses of fixed-schedules suramin treatment in patients with newly diagnosed metastatic prostate cancer: a southwest oncology group study. $J$ Clin Oncol 2000 mar; 18 (5): 1043-1049.

46. BOISIER S, MAGNETTO S, FRAPPART L et al.: Bisphosphonates inhibit prostate cancer and breast carcinoma cell adhesion to unmineralized and mineralized bone extracellular matrices. Cancer Res $1997 \mathrm{sep}$ 15; 57 (18): 3890-3894.

47. COLEMAN RE, WOLL PJ, MILES M et al.: Treatment of bone metastases from breast cancer with 3-amino-1, 1 hydroxypropylidene bisphosphonate (APD). $\mathrm{Br} J$ Cancer 1988; 56: 465-469.

48. MORTON AR, CANTRILL JA, PILLAI GV, MCMAHON A, ANDERSON DC, HOWELL A.: Sclerosis of lytic bone metastases after disodium aminohydroxypropylidene bisphosphonate (APD) in patients with breast cancer. Br Med J 1988; 297: 772-773.

49. VAN HOLTEN-VERZANTVOORT AT, KROON HM et al.: Palliative bone treatment in patients with bone metastases from breast cancer. J Clin Oncol 1993; 11: 491498.

50. POLLARD M, LUCKERT PM.: Effects of dichloromethylene diphosphonate on the osteolytic and osteoblastics effects of rate prostate adenocarcinoma cells. $J$ Natl Cancer Inst USA 1985; 75: 949-951.

51. POLLARD M, LUCKERT PM.: The beneficial effects of diphosphonate and piroxicam on the osteolytic and metastatic spread of rat prostate carcinoma cells. Prostate 1986; 8: 81-86.
52. POLLARD M, LUCKERT PM, SCHEU J.: Effects of diphosphonate and X-rays on bone lesions induced in rats by prostate cancer cells. Cancer 1988; 61: 20272032.

53. NEMOTO R, KANOH S, KOISO K et al.: Establishment of a model to evaluate inhibition of bone resorption induced by human prostate cancer cells in nude mice. J Urol 1988; 140: 875-879.

54. CLARKE NW, HOLBROOK JB, McCLURE J, GEORGE NJR.: Osteoclast inhibition by pamidronate in metastatic prostate cancer: a preliminary study. $\mathrm{Br} J$ Cancer 1991; 63: 420-423.

55. ADAMI S, MIAN M.: Clodronate therapy of metastatic bone disease in patients with prostatic cancer. In: bisphosphonates and tumor osteolysis. Brunner KW, Fleish H, Senn HJ. Eds. Springer. Heidelberg: 67-72.

56. PUROHIT OP, ANTHONY CR, RADSTONE CR, OWEN J, COLEMAN RE.: High-dose intravenous pamidronate for metastatic bone pain. Br J Cancer 1994; 70: 554558.

57. LIPTON A, GLOVER D, HARVEY H et al.: Pamidronate in the treatment of bone metastases: results of 2 doseranging trials in patients with breast or prostate cancer. Ann Oncol 1994; 5 (suppl 7): 531-535.

58. HARVEY HA, LIPTON A.: The role of bisphosphonates in the treatment of bone metastases-the us experience support care. Cancer 1996; 4: 213-217.

59. VORREUTHER R.: Bisphosphonates as an adjunct to palliative therapy of bone metastases from prostatic carcinoma a pilot study on clodronate. Br J Urol 1993; 72: 792-795.

60. KYLMALA T, TAMINELA TL, LINDHOLM TS et al.: The effect of combined intravenous and oral clodronate on bone pain in patients with metastatic prostate cancer. Ann Chir Gynaecol 1994; 83: 316-319.

61. CRESSWELL S, ENGLISH PJ, HALL RR et al.: Pain relief and quality of life assessment following intravenous and oral clodronate in hormone escaped metastatic prostate cancer. Br J Urol 1995; 76: 360365.

62. SASAKI A, BOYCE BF, STORY B et al.: Bisphosphonate risedronate reduces metastatic human breast cancer burden in bone in nude mice. Cancer Res 1995; 55: 3551-3557.

63. BEN-JOSEF E, PORTER AT.: Radioisotopes in the treatment of bone metastases. Ann Med 1997 feb; 29 (1): 31-35.

64. MCEWAN AJ.: Use of radionuclides for the palliation of bone metastases. Semin Radiat Oncol 2000 apr; 10 (2): 103-114.

65. ROBINSON RG, BLAKE GM, PRESTON DF et al.: Stromtium-89: treatment results and kinetics in patients with painful metastatic prostate and breast cancer in bone. Radiographics 1989; 9: 271.

66. LEWINGTON VJ, MCEWAN AJ, ACKERY DM et al.: A prospective, randomised double-blind crossover study to examine the efficacy of stromtium-89 in pain palliation in patients with advanced prostate cancer metastatic to bone. Eur $J$ Cancer 1991; 27: 954.

67. CARROLL PR, SUGIMURA K, COHEN MB et al.: Detection and staging of prostate carcinoma after transurethral resection or open enucleation of the prostate: accuracy magnetic resonance imaging. J Urol 1992; 147: 402 .

68. LAMB HM, FAULDS D.: Samarium 153sm lexidronam. Drugs Aging 1997 nov; 11 (5): 413-418. 
69. SERAFINI AN, HOUSTON SJ, RESCHE I et al.: Palliation of pain associated with metastatic bone cancer using samarium-153 lexidronam: a double-blind placebo-controlled clinical trial. J Clin Oncol 1998 apr; 16 (4): 1574-1581.

70. TIAN JH, ZHANG JM, HOU QT et al.: Multicentre trial on the efficacy and toxicity of single-dose samarium153-ethylene diamine tetramethylene phosphonate as a palliative treatment for painful skeletal metastases in china. Eur J Nucl Med 1999 jan; 26 (1): 2-7.

71. LIMOURIS G, SHUKLA SK, MANETOU A et al.: Rhenium-186-HEDP palliative treatment in disseminated bone metastases due to prostate cancer. Anticancer Res 1997 may-jun; 17 (3B): 1699-1704.

72. GRAHAM MC, SCHER HI, LIU GB et al.: Rhenium186-labeled hydroxyethylidene diphosphonate dosimetry and dosing guidelines for the palliation of skeletal metastases from androgen-independent prostate cancer. Clin Cancer Res 1999 jun; 5 (6): 1307-1318.

73. DE KLERK JM, ZONNENBERG BA, KROUWER HG et al.: Transient cranial neuropathy in prostatic cancer with bone metastases after rhenium-186-HEDP treatment. J Nucl Med 1996 mar; 37 (3): 465-467.

74. GRANT R, PAPADOPOULOS SM, GREENBERG HS.: Metastatic epidural spinal cord compression. Neurol Clin 1991; 9 (4): 825.

75. BRIDWELL KH.: Treatment of metastatic prostate cancer of the spine. Urol Clin North Am 1991; 18 (1): 153

76. RABBANI SA.: Metalloproteases and urokinase in angiogenesis and tumor progression in vivo 1998 janfeb; 12 (1): 135-142.

77. FESTUCCIA C, GUERRA F, D'ASCENZO S, GIUNCIUGLIO D, ALBANI A, BOLOGNA M.: In vitro regulation of pericellular proteolysis in prostatic tumor cells treated with bombesin. Int $J$ Cancer 1998 jan 30; 75 (3): 418-431.

78. STEARNS ME, RHIM J, WANG M.: Interleukin 10 (IL10) inhibition of primary human prostate cell-induced angiogenesis: IL-10 stimulation of tissue inhibitor of metalloproteinase- 1 and inhibition of matrix metalloproteinase MMP-2/MMP-9 secretion. Clin Cancer Res 1999 jan; 5 (1): 189-196.
79. LOKESHWAR BL.: MMP inhibition in prostate cancer. Ann Ny Acad Sci 1999 jun 30; 878: 271-289.

80. WANG KH.: The genetic and molecular basis for chemoprevention: head and neck cancer as a model ASTRO's 41 annual meeting. San Antonio, Texas 1999 october 31-november 4: 35.

81. CULINE S, KRAMAR A, DROZ JP, THEODORE C.: Phase I study of all-trans retinoic acid administered intermittently for hormone refractory prostate cancer. J Urol 1999 jan; 161 (1): 173-175.

82. RITCHIE CK, THOMAS KG, ANDREWS LR, TINDALL DJ, FITZPATRICK LA.: Effects of the calciotrophic peptides calcitonin and parathyroid hormone on prostate cancer growth and chemotaxis. Prostate 1997 feb 15; 30 (3): 183-187.

83. SLOVIN SF, SCHER HI, DIVGI CR et al.: Interferongamma and monoclonal antibody 131I-labeled CC49: outcomes in patients with androgen-independent prostate cancer. Clin Cancer Res 1998 mar; 4 (3): 643651.

84. SINHA AA, QUAST BJ, REDDY PK, ELSON MK, WILSON MJ.: Intravenous injection of an inmunoconjugate (anti-PSA-Ig conjugated to 5-fluoro-2-deoxyuridine) selectively inhibits cell proliferation and induces cell death in human prostate cancer cell tumor grown in nude mice. Anticancer Res 1999 mar-apr; 19 (2a): 893-902.

85. OLAPADE-OLAOPA EO, MOSCATELLO DK, MACKAY $\mathrm{EH}$ et al.: Evidence for the differential expression of a variant EGF receptor protein in human prostate cancer. Br J Cancer 200 jan; 82 (1): 186-194.

Dra. M. Rodríguez Cordón

C/ Guardia Civil, 23 - Esc. 3, pta. 19

46020 Valencia

(Trabajo recibido el 20 octubre de 2000)

\section{COMENTARIO EDITORIAL}

En primer lugar reconocer y felicitar a los autores por la amplia revisión de la literatura realizada. De la misma, nos aportan una síntesis actualizada, exponiéndonos todas las posibles alternativas terapéuticas disponibles hoy en día para el carcinoma de próstata diseminado, constituyendo así un trabajo muy útil para el urólogo que a diario tiene que tratar este tipo de pacientes.

Estando de acuerdo con las opciones enumeradas de tratamiento asi como con el carácter multidisciplinar que éste debe tener, involucrando a distintos especialistas (Urólogo, Radio- terapeuta, Médicos de la Unidad del Dolor, etc...) quisiera añadir algunas consideraciones acerca del manejo del paciente en la práctica clínica. Como refieren los autores, son varios y distintos los tratamientos que se precisan en esta fase de la enfermedad, pero el paciente no debería percibirlos como una simple suma de tratamientos (con efectos secundarios nada despreciables) y de consultas por distintos especialistas que acaba de conocer (justo en los momentos en que se encuentra peor), sino que debemos intentar un tratamiento integral y bien coordinado, siendo deseable que cada grupo de trabajo tenga bien definidos los pasos a seguir. 
En primer lugar, es importante diferenciar claramente entre el tratamiento oncológico y el tratamiento analgésico de la enfermedad, pues los problemas y los responsables de uno y otro son bien distintos.

Así, del tratamiento oncológico, basado fundamentalmente en las manipulaciones hormonales, el máximo responsable debe ser el Urólogo, quien decidirá cuándo y con qué realiza el bloqueo hormonal, asi como los criterios para sus cambios, contando con la información precisa para que el paciente conozca el tratamiento estándar asi como las alternativas, como el bloqueo intermitente, y del que, fuera de ensayos clínicos, debemos explicar bien las ventajas y riesgos de su elección. Los autores revisan en el trabajo esta opción señalando la falta de resultados sobre la supervivencia. También nos hacen referencia sobre la controversia existente acerca del bloqueo androgénico combinado, especificando los pacientes que más parecen beneficiarse del mismo. Aunque esta discusión sigue abierta, en caso de realizarlo, y aunque existen diversos fármacos antiandrógenos (esteroideos y no esteroideos, estrógenos), creo que existe un amplio consenso en que el fármaco a añadir al análogo LH$\mathrm{RH}$, debe ser un antiandrógeno no esteroideo como la flutamida o la bicalutamida.

De otro lado, cuando aparece el dolor, generalmente óseo, es necesario instaurar el tratamiento analgésico, siendo los responsables de su elección y aplicación, además del Urólogo, los especialistas correspondientes (analgésicos por la Unidad del Dolor, RT por radioterapeutas, Sr-89 por Medicina Nuclear, etc.), adquiriendo asi el carácter de tratamiento multidisciplinar. No obstante, la coordinación de los diferentes tratamientos y el seguimiento debe continuar a cargo del Urólogo, pues suele ser en quien el paciente confía, acaso por conocerle desde el inicio de la enfermedad, y quien le resolverá las complicacio- nes urológicas que pueda presentar (sondajes, RTU desobstructiva, colocación de doble J, nefrostomías, etc.).

Nuestro grupo de trabajo es desde hace años defensor de esta forma de actuación ${ }^{1}$, asi como de utilizar el arsenal terapéutico disponible y tan sólidamente expuesto en esta revisión, de una forma escalonada, siguiendo las recomendaciones de la O.M.S. ${ }^{2}$, desde el uso de los AINEs, de elección en el dolor óseo leve por su efecto anti-inflamatorio (primer escalón), pasando a los opiáceos menores y coanalgésicos (corticoides y antidepresivos) si el dolor es moderado o fracasan aquellos ( $2^{\circ}$ escalón), y si persiste, utilizar la radioterapia (RT) local si el dolor óseo es localizado, o radiofármacos (Sr-89) si el dolor óseo es multifocal, o bisfosfonatos (zoledronato), no teniendo experiencia con la RT hemicorporal, a la que creemos en desuso por sus importantes efectos secundarios.

Los resultados que nos refieren los autores con la RT local, el Sr-89, o ambos, son alentadores. En nuestra experiencia con el $\mathrm{Sr}-89^{3}$ observamos que en su indicación precoz la eficacia era significativamente mayor que cuando se utilizaba en las fases finales con dolor óseo severo (tercer escalón), en donde sólo tendrán cabida los mórficos mayores, bien por vía transdérmica o espinal.

\section{REFERENCIAS}

1. CÓZAR JM, GÁlVEZ R, TALLADA M.: Modalidades terapéuticas del dolor óseo en el cáncer de próstata. Actas Urol Esp 1999; 23 (5): 464-470.

2. JADDAD AR, BROWMAN GP.: The WHO analgesic ladder for cancer pain management. JAMA 1995; 274 (23): 1870-1881.

3. CÓZAR JM, TALLADA M, GÁlVEZ R y cols.: Tratamiento con estroncio-89 del dolor óseo metastásico por carcinoma de próstata. Nuestra casuística. Urol Integr Invest 1998; 3: 330-334.

Fdo.: José M. Cózar Olmo

Servicio de Urología

Hospital Univ. Virgen de las Nieves

Granada 